\title{
The Cold Gas System on TDX - Accurate in-Orbit Evaluation
}

\author{
Daniel Schulze ${ }^{1}$, Ralph Kahle ${ }^{2}$, Jaap Herman ${ }^{3}$ \\ German Aerospace Center (DLR), Oberpfaffenhofen, 82234 Wessling, Germany \\ Arno Dietrich ${ }^{4}$ \\ Airbus Defence \& Space, 88039 Friedrichshafen, Germany
}

\begin{abstract}
The TanDEM-X mission comprises two nearly identical satellites - TSX and TDX that fly at an altitude of $\sim 515 \mathrm{~km}$ in a close formation at distances down to $120 \mathrm{~m}$. The standard orbit corrections are made with hydrazine propulsion on both spacecraft, but the relative geometry is maintained by a dedicated cold gas system on TDX only, which initially comprised $36 \mathrm{~kg}$ of nitrogen. The routine formation-keeping requires at least one pair of cold gas maneuvers daily.
\end{abstract}

Formation flight started in late 2010 and continued ever since in several configurations. This means that by now of the order of 2500 cold gas maneuvers have been performed and the remaining amount of fuel will allow for another 1500 to be made. Maneuvers were done mainly with the four $\mathbf{4 0}$ milli-Newton thrusters of branchA, but about $25 \%$ of the time branch-B was used.

The construction of a digital elevation model of the Earth requires very precise orbit information, which is delivered by the flight dynamics department at GSOC. This also yields an accuracy of $<0.1 \mathrm{~mm} / \mathrm{s}$ in the a posteriori reconstruction of maneuvers.

The large number of maneuvers and the extremely accurate orbit information allow a unique in-orbit evaluation of the cold gas system. Thruster performance is reconstructed from the precise orbit determination combined with $1 \mathrm{~Hz}$ telemetry during the burn and a priori laboratory measurements. Among others comparisons are made between the two branches and between maneuvers in flight- and anti-flight direction. The influence of the maneuver duration and the tank pressure will also be presented.

\section{Introduction}

$\mathrm{T}$ HE TanDEM-X project is implemented in a "Public-Private Partnership" between the German Aerospace Centre and Astrium GmbH.

The main objective of the TanDEM-X (TerraSAR-X add-on for Digital Elevation Measurement) mission is to create a global digital elevation model with $12 \mathrm{~m}$ horizontal and $2 \mathrm{~m}$ vertical resolution [1]. To achieve this, two satellites - TerraSAR-X (TSX) and TanDEM-X (TDX; a satellite of almost identical construction) are forming the first configurable SAR (Synthetic Aperture Radar) interferometer in space with a separation of a few hundred meters only. A powerful ground segment, which is interlaced with that of TSX, completes the TanDEM-X system. The satellites are currently flying in a close formation with cross-track distances down to $120 \mathrm{~m}$. Three years of formation flying with various baselines are necessary to collect sufficient measurements for the generation of the global Digital Elevation Model (DEM).

1 AOCS engineer, German Space Operations Center, Oberpfaffenhofen, 82234 Wessling, Germany/ D.Schulze@dlr.de

${ }^{2}$ Flight Dynamics engineer, German Space Operations Center, Oberpfaffenhofen, 82234 Wessling, Germany/ Ralph.Kahle@dlr.de

3 AOCS team lead, German Space Operations Center, Oberpfaffenhofen, 82234 Wessling, Germany/ Jaap.Herman@dlr.de

4 System engineer, Airbus Defence \& Space, Friedrichshafen, 88039 Friedrichshafen, Germany/ Arno.Dietrich@ASTRIUM.EADS.NET 
DLR is responsible for the scientific exploitation of the TSX/TDX data, as well as for the planning and implementation of the mission, for controlling the two satellites and for generating the digital elevation model. Astrium Satellites* has built the satellites and shares in the costs of development and exploitation. The responsibility for marketing the data lies in the hands of Infoterra $\mathrm{GmbH}$, a subsidiary of Astrium $\mathrm{GmbH}$, as was the case already with TSX data.

TSX was launched from Baikonur, Kazakhstan, on June $15^{\text {th }} 2007$ with a Dnepr rocket into a sun-synchronous dusk-dawn orbit at an altitude of $514 \mathrm{~km}$. The ground coverage is repeated in an eleven day cycle which is achieved by controlling the spacecraft in a $\pm 250 \mathrm{~m}$ tube around a predefined Earth-fixed reference orbit [2].

TDX was also launched from Baikonur almost exactly three years after TSX, namely on June $21^{\text {st }} 2010$. The initial distance of $16000 \mathrm{~km}$ towards TSX was soon reduced, and a far formation at $20 \mathrm{~km}$ along-track separation was acquired within 30 days after launch. The first close formation was reached by mid of October and the DEM acquisition started in December 2010 with an intersatellite distance of $\sim 300 \mathrm{~m}$ (see [3] for details on the formation acquisition process).

The TDX satellite is in general a soft- and hardware rebuild of TSX, but some changes were made in order to allow for close formation flight. The most important ones are the cold gas propulsion system and a software module for experimental autonomous formation flight (TAFF).

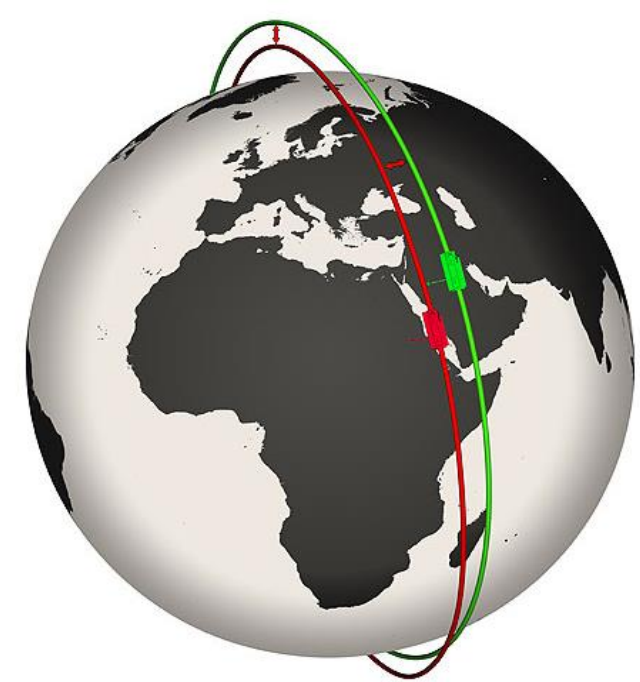

Figure 1. TSX and TDX flying in close formation. TDX (green) describes a helix around the orbit of TSX (red). The radial separation is largest over the poles and becomes zero at the equator. Here the separation is maximal in normal direction. The distance between the satellites is typically between 150 and 500 meters. [Source: www.dlr.de]

\section{A. Formation Flight}

The close formation of TSX and TDX is dictated by the wish for a configurable SAR interferometer in space. The satellites fly in almost identical orbits whereby the trajectory of TDX describes a helix around that of TSX. This is achieved by the concept of relative eccentricity and inclination vector separation [4]. The maximal radial separation is reached over the poles (vertical baseline typically between $200 \mathrm{~m}$ and $500 \mathrm{~m}$ ) and the maximum separation in normal direction occurs at the equator (horizontal baseline typically $200-500 \mathrm{~m}$; see Fig. 1). Thus it is guaranteed that the radial and normal separation never become zero at the same time. The details of the formation depend upon the mission phase. The one with the smallest baseline had a minimum cross-track separation of $120 \mathrm{~m}$.

The TDX-TSX relative motion is perturbed by external and internal forces. The dominating source is the oblateness of the Earth, which perturbs the slightly different eccentricity vectors of TSX and TDX in different ways, resulting in a tilt of the relative motion ellipse. Furthermore, the slightly different atmospheric drag as well as the relative (along-track) maneuver errors result in a change of the relative semi-major axis, which then yields an along-track drift. An illustration of the TDX-TSX relative motion and control can be found in [5].

The relative orbit perturbations need to be compensated by in-plane formation keeping maneuvers to maintain a stable configuration. For example, a formation with a vertical separation of $300 \mathrm{~m}$ requires every day two burns of approximately $0.5 \mathrm{~cm} / \mathrm{s}$ each which are separated by half an orbit. These maneuvers primarily serve for the correction of the relative eccentricity vector, but they are used to adjust the along-track separation and to compensate possible differential drag effects, too. They are exclusively performed on TDX with the cold gas system.

\section{B. The cold gas system on TDX}

The cold gas system on TDX has two redundant branches with four $40 \mathrm{mN}$ thrusters each. Two are mounted on the $+\mathrm{x}$ (body frame) side and two on the $-\mathrm{x}$ side. This corresponds to the flight-direction $(+\mathrm{x})$ and anti-flight

*Company changed its name to Airbus Defence \& Space 


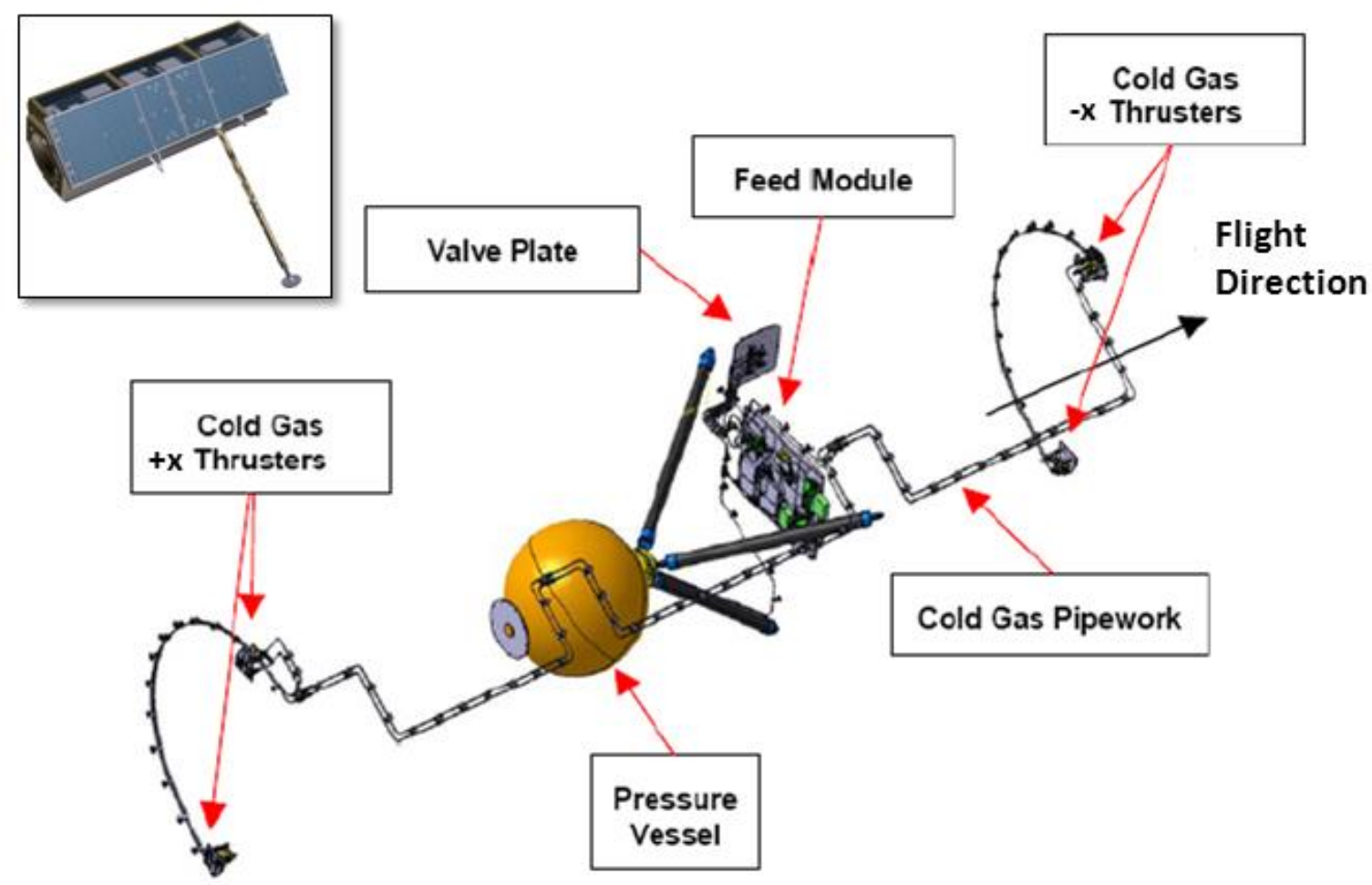

Figure 2. Illustration of the cold gas system. The pressure vessel is shown in yellow. Two thruster pairs are mounted in flight- and in anti-flight direction each. Most cold gas components are accommodated on the feed module. [Source: Astrium Satellites]

direction (-x) when the satellite has its nominal attitude for routine operations. The cold gas system consists of a high pressure and a low pressure section (Fig. 2). The pressure vessel contained $36.4 \mathrm{~kg}$ of gaseous nitrogen (GN2) at launch and is connected by a pipework to the feed module. This is a plate that accommodates most of the cold gas components, such as the low and high pressure transducers (LPT \& HPT), low and high pressure filters (LPF \& HPF), high pressure latch valves (HPLV) and pressure regulators (PR) together with their interconnecting pipework. The valve plate contains the fill and vent valve, the test ports and the relief valve. The high pressure at launch was 280 bar. Each branch can be closed and opened by its own HPLV.

Both branches were successfully tested on ground and during the LEOP of TDX. It was decided to use branch B as prime, because it had shown a more stable performance in ground tests. In September 2011 however, after eleven months of formation flight, a switch to branch A had to be made, because of an unusual behavior of the prime branch (cf. [5] for details). Although investigations have shown that branch B is still operational, it was decided to continue the formation flight with branch A as operational prime.

\section{Maneuver calibration and reconstruction}

There are two methods to reconstruct the size of the cold gas maneuvers that are executed on-board of TDX. One approach is based upon the specified thrust constants in combination with the commanded on-time and $1 \mathrm{~Hz}$ telemetry of the low pressure transducer. This method will be called book-keeping method in the following. The generated thrust as a function of the inlet pressure can be described by the linear expression $F(p)=a+b p$, where $p$ is the low pressure and the coefficients $a$ and $b$ are specific for each thruster. It is accumulated over the burn duration $\Delta t$ and all active thrusters (summation index $i$ ). The velocity increment can be derived by

$$
\Delta v=\frac{\sum_{i}\left(\boldsymbol{a}_{i}+\boldsymbol{b}_{i} \overline{\boldsymbol{p}}\right) \Delta \boldsymbol{t}}{\boldsymbol{M}_{S C}}
$$

with $\bar{p}$ the average low pressure during the burn and $M_{S C}$ the spacecraft's mass. The coefficients $a$ and $b$ have been determined by the manufacturer and are based on an analytical model and verification measurements. Uncertainties in the coefficients are of the order of $5 \%$. 
Temperature variations are not included in Eq. (1). They are small due to the active thermal control of the satellite and the sun-synchronous orbit. The thruster brackets are controlled within a temperature of $20 \pm 0.5^{\circ} \mathrm{C}$. Temperature variations in the other CGS components are smaller than $5^{\circ} \mathrm{C}$.

The second way of maneuver reconstruction is done by means of a precise orbit determination (POD). POD is carried out by GSOC on a routine basis using measurements of the IGOR GPS receiver. The process is based on a reduced dynamic orbit determination using the "GPS High precision Orbit determination Software Tools" (GHOST), which was developed at GSOC in cooperation with Delft University of Technology. See [6] for a general description or [7] for details on the employed physical models and the operational implementation.

The GSOC POD maneuver calibration was first used in the GRACE mission. GRACE also has $40 \mathrm{mN}$ cold gas thrusters and the calibrated maneuvers range from maneuver sizes between 1 and $12 \mathrm{~cm} / \mathrm{s}$. The overall accuracy of calibrations based upon external orbit comparison is of the order of $1 \%$ [8].

The size of typical TDX formation-keeping maneuvers is 0.4 to $0.7 \mathrm{~cm} / \mathrm{s}$ only, which implies a somewhat larger uncertainty. In order to estimate the POD calibration performance, small artificial maneuvers were inserted into the POD processing of different TDX flight data arcs. While the POD calibrated the true maneuvers with a similar performance as compared to the original POD process, the artificial (i.e. $0 \mathrm{~cm} / \mathrm{s}$ ) maneuvers were estimated with very small size of $0.006 \mathrm{~cm} / \mathrm{s}$ on average. This means that on TDX the accuracy will be $1.5-2.5 \%$ for the typical maneuver sizes mentioned above.
Table 1. Thruster coefficients used in the book-keeping method.

\begin{tabular}{|c|c|c|}
\hline Thruster & $\begin{array}{c}\text { Offset } \\
\boldsymbol{a}[\mathrm{mN}]\end{array}$ & $\begin{array}{c}\text { Slope } \\
\boldsymbol{b}[\mathrm{mN} / \mathrm{bar}]\end{array}$ \\
\hline A1-plus & 1.54 & 26.666 \\
\hline A1-minus & 1.577 & 26.666 \\
\hline A2-plus & 1.416 & 26.666 \\
\hline A2-minus & 1.312 & 26.666 \\
\hline B1-plus & 2.685 & 26.666 \\
\hline B1-minus & 1.811 & 26.666 \\
\hline B2-plus & 1.766 & 26.666 \\
\hline B2-minus & 1.682 & 26.666 \\
\hline
\end{tabular}

\footnotetext{
* For the purpose of POD and occultation measurements, TerraSAR-X and TanDEM-X carry the Integrated Geodetic Occultation and Ranging (IGOR) receiver. IGOR was developed by Broad Reach Engineering and provided by the German Research Centre for Geosciences (GFZ). IGOR is a geodetic grade dual-frequency receiver with 12 channels allocated for the tracking of GPS satellites for navigation.
} 


\section{Results}

\section{A. Pressure profile}

\section{Pressure profile}

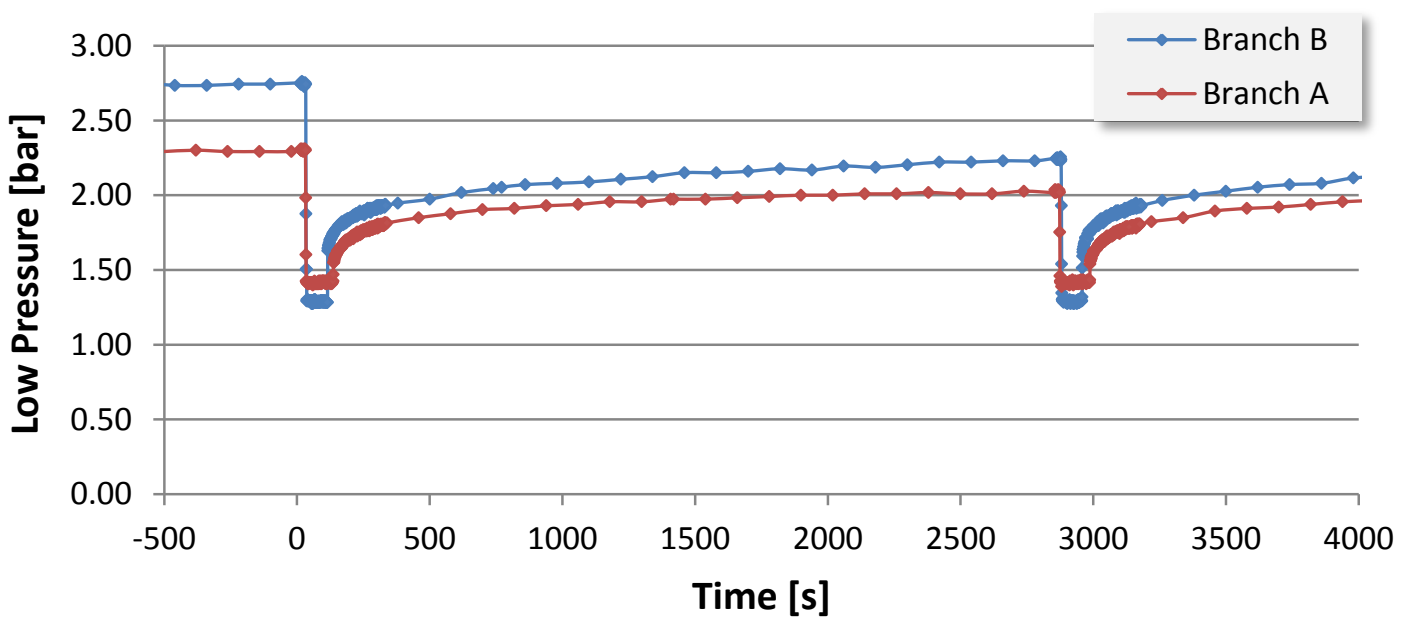

Figure 3. Pressure profiles. The maneuvers with branch $B$ were made on $2011 / 05 / 25$, those with branch A on 2012/06/01. The zero-point of the abscissa marks the start of the first burn of both pairs. The second maneuver starts after half an orbit (47 minutes). The initial pressure is lower than at the first burn and the difference is larger for branch B. Both pressure regulators work as expected and known from ground tests.

Figure 3 shows the pressure profiles of two maneuvers pairs. The first one was made on 2011/05/25 with branch B, the second on 2012/06/01 with branch A. The pressure regulators show slightly different values during the burn within the specification of $1.5 \pm 0.4$ bar. The pressure drops from 2.3 to 1.4 bar on branch A and from 2.75 bar to 1.3 bar on branch B. It stabilizes within roughly five seconds at the value observed during the burn. The pressure increases again after the burn and reaches its previous level after about 12 hours. The second maneuver normally follows already after half an orbit i.e. after 47 minutes. It can be seen in Fig. 3 that the initial pressure of the second burn is therefore smaller than for the first. The difference in the example is 0.23 bar for branch A and 0.5 bar for branch B. This also affects the pressure average of the burn (see table 2). The described behavior of PR-A and PR-B was already seen in ground tests.

Table 2. Example of four typical maneuver pairs. The second maneuver follows half an orbit after the first one and is made in the opposite direction. The pressure has not yet fully recovered and the second maneuver has a lower average pressure.

\begin{tabular}{|l|c|c|c|c|c|c|}
\hline $\begin{array}{c}\text { Thruster } \\
\text { pair }\end{array}$ & Date & $\begin{array}{c}\text { Burn } \\
\text { Duration }[\mathrm{s}]\end{array}$ & $\begin{array}{c}p_{-} \text {start } \\
{[\mathrm{bar}]}\end{array}$ & $\bar{p}[\mathrm{bar}]$ & $\begin{array}{c}\Delta v[\mathrm{~cm} / \mathrm{s}] \\
\text { by BK }\end{array}$ & $\begin{array}{c}\Delta v[\mathrm{~cm} / \mathrm{s}] \\
\text { by POD }\end{array}$ \\
\hline A-minus & $2012 / 05 / 3120: 42: 02$ & 113.194 & 2.31 & 1.448 & -0.683 & -0.681 \\
\hline A-plus & $2012 / 05 / 3121: 29: 32$ & 105.627 & 2.03 & 1.439 & 0.636 & 0.640 \\
\hline A-plus & $2012 / 06 / 0119: 36: 49$ & 104.454 & 2.29 & 1.449 & 0.633 & 0.633 \\
\hline A-minus & $2012 / 06 / 0120: 24: 10$ & 113.619 & 2.00 & 1.437 & -0.680 & -0.681 \\
\hline B-plus & $2011 / 05 / 2418: 01: 34$ & 80.928 & 2.77 & 1.376 & 0.472 & 0.451 \\
\hline B-minus & $2011 / 05 / 2418: 48: 58$ & 84.429 & 2.24 & 1.331 & -0.470 & -0.458 \\
\hline B-plus & $2011 / 05 / 2516: 09: 42$ & 80.000 & 2.75 & 1.375 & 0.466 & 0.444 \\
\hline B-minus & $2011 / 05 / 2516: 57: 09$ & 78.576 & 2.25 & 1.337 & -0.440 & -0.427 \\
\hline
\end{tabular}




\section{B. Regulated Pressure}

Figure 4 shows the regulated pressure as a function of mission elapsed time (MET). Again the average pressure during the burn is used. The initial value for the first burn is larger than for the second burn of each maneuver pair (cf. II.A). This is also seen in the average pressure in Fig. 4. The values of the first maneuver are separated from the second one by $\sim 0.01$ bar for branch A and by $\sim 0.035$ bar for branch B.

PR-A (red diamonds) delivers increasing pressure with decreasing tank pressure (green line). Starting at 1.4 bar it reaches 1.5 bar around MET 1300. The dependency upon the inlet pressure was already seen in ground performance tests. The effect is not observed for PR-B however (blue triangles). A peak is seen at around MET $=730$. The maneuvers on those days were planned autonomously by TanDEM-X Autonomous Formation Flying Experiment (TAFF) and have much smaller burn durations (for details on TAFF please refer to [9]). Short burn duration leads to a higher pressure average during the maneuver, because the drop to the floor pressure takes a few seconds. Another peak is visible at MET 278 for branch B. It also stems from another TAFF closed loop campaign but comprises fewer maneuvers than the second campaign at MET 750.

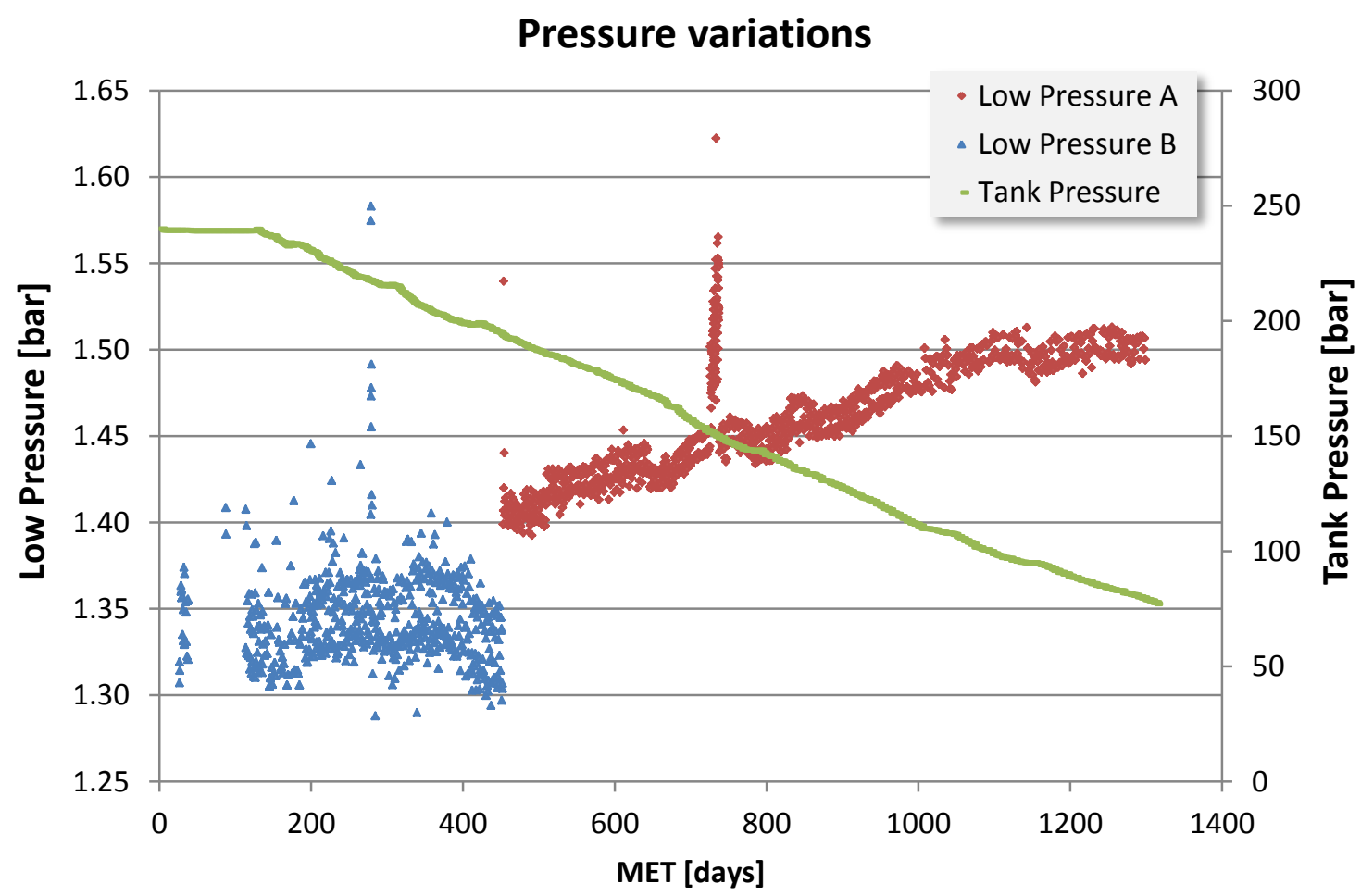

Figure 4. Pressure variations over mission elapsed time (MET). PR-A (red squares) is dependent upon the tank pressure (green line). The second maneuver of each pair has a smaller average pressure than the first one. The peaks at MET 278 and 750 indicate maneuvers autonomously planned by the TAFF module.

\section{Evaluation of performance}

A total of 2360 cold gas maneuvers were made between 2010-07-20 and 2014-01-14 and are included in the analysis. The distribution over the different thruster pairs is shown in Fig. 5. 98 maneuvers were planned autonomously by the TAFF module and have been excluded from the analysis.

Figure 6 shows the deviations of the two calibration methods described in section I.C. A new parameter $\Delta \mathrm{cal}$ is introduced here, describing the deviation of both calibration methods as $\Delta \mathrm{cal}=\left(\Delta \mathrm{v}_{\mathrm{POD}}-\Delta \mathrm{v}_{\mathrm{BK}}\right) / \Delta \mathrm{v}_{\mathrm{BK}}$.

\section{Maneuver Count}

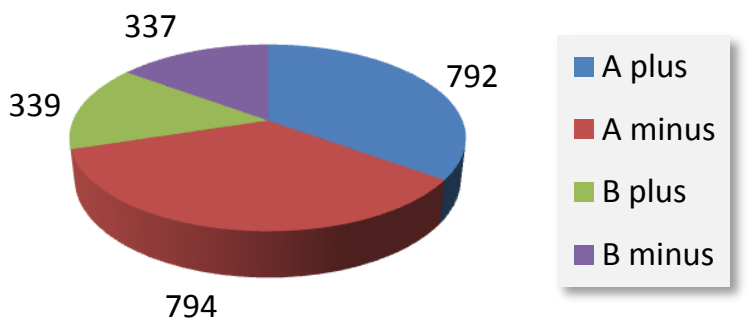

Figure 5: Number of maneuvers per thruster pair. 


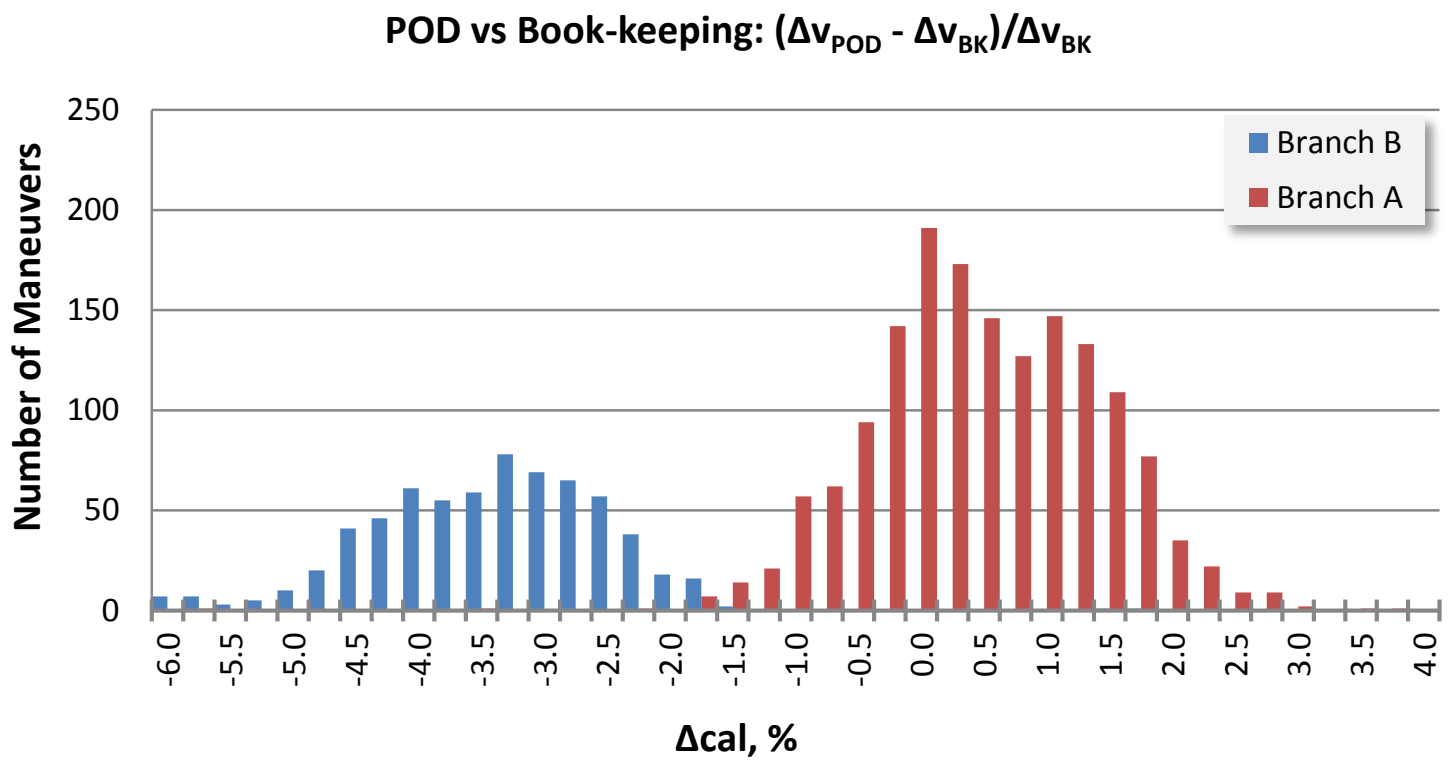

Figure 6: POD vs book-keeping histogram. Shown is the difference between the $\triangle v$ derived by POD and by the book-keeping method. Not included are TAFF maneuvers. The statistic average values $-3.40 \pm 1.38 \%$ for branch $B$ and $0.61 \pm 0.96 \%$ for branch $A$.

The mean $\Delta \mathrm{cal}$ for branch A is $0.6 \%$ with a standard deviation of $1.0 \%$. Branch B shows a mean $\Delta \mathrm{cal}$ of $3.4 \%$ with a $\sigma$ of $1.4 \%$. A small mean difference implies that POD and book-keeping match well and the POD results are in good agreement with the a priori laboratory measurements. Note that a constant difference between POD and book-keeping is taken into account in the planning of maneuvers. The accuracy of the POD method was estimated in section I.C to be $<2.5 \%$ depending upon maneuver size. The actually measured standard deviations confirm this and also show that the random errors in the book-keeping method are comparable. A further discussion of this result will be done in chapter II.D.

Figure 7 shows the dependency of $\Delta$ cal upon the average pressure during the burn. Two trends are visible: For branch A $\Delta \mathrm{cal}$ is $>0$ and grows with increasing pressure. For branch B, however, it is decreasing with increasing pressure. The reason for this behavior must lie within the book-keeping method, because the calibration by POD is not depending upon the pressure at all. This suggests that the coefficients as measured on ground and used in Eq. (1) for the book-keeping method are different in orbit.

Figure 8 shows the dependency of $\Delta$ cal upon the burn duration. The standard deviation is larger for shorter maneuvers. This is most obvious in the data from branch $\mathrm{A}$, because it comprises more maneuvers. The standard deviation for branch A is $0.93 \%$ for burn durations between 75 and 100 seconds (778 maneuvers), decreases to $0.68 \%$ for on-times between 100 and 125 seconds (340 maneuvers) and reaches $0.53 \%$ for the longest maneuvers between 125 and 200 seconds duration (128 maneuvers). This trend is caused by increasing relative errors in both calibration methods for smaller burn durations. The mean absolute error in the POD is $0.006 \mathrm{~cm} / \mathrm{s}$ (see I.C) and does not depend upon maneuver size. The relative error in the book-keeping method is increasing, because the fixed telemetry rate of $1 \mathrm{~Hz}$ does not provide sufficient data samples for a good average function. It is interesting that virtually all burns with duration $<75$ seconds on branch A show a $\Delta$ cal $>0$. This is not yet fully understood, but a possible explanation could be that these maneuvers were all made later in the mission when the inter satellite distance was particular small. As described in II.A, the regulated pressure is increasing with decreasing tank pressure and therefore also with MET (see Fig. 5). The average pressure is related to the $\Delta \mathrm{cal}$ as seen in Fig. 7 and thus could be the root cause for this observation. All maneuvers with burn duration $<75$ seconds were made with an average pressure above 1.48 bar.

The results of the different thruster pairs are shown in Fig. 11-14 in the appendix. TAFF maneuvers are not included in the current analysis. 


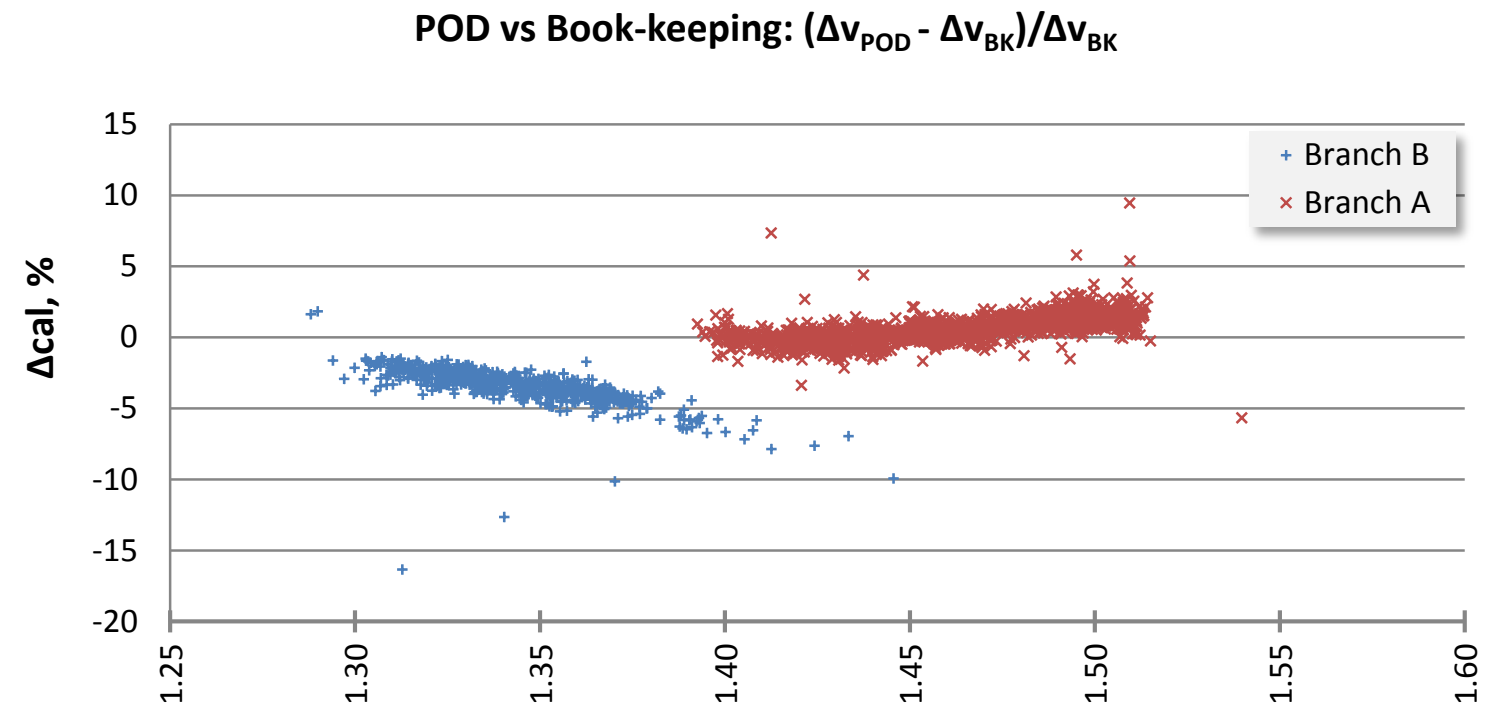

Low Pressure, bar

Figure 7: Dependency upon low-pressure. $\Delta$ cal for branch $A$ is increasing with average pressure, whereas it is decreasing for branch $B$. This arises from the book-keeping method because the calibration via POD is not dependent upon pressure.

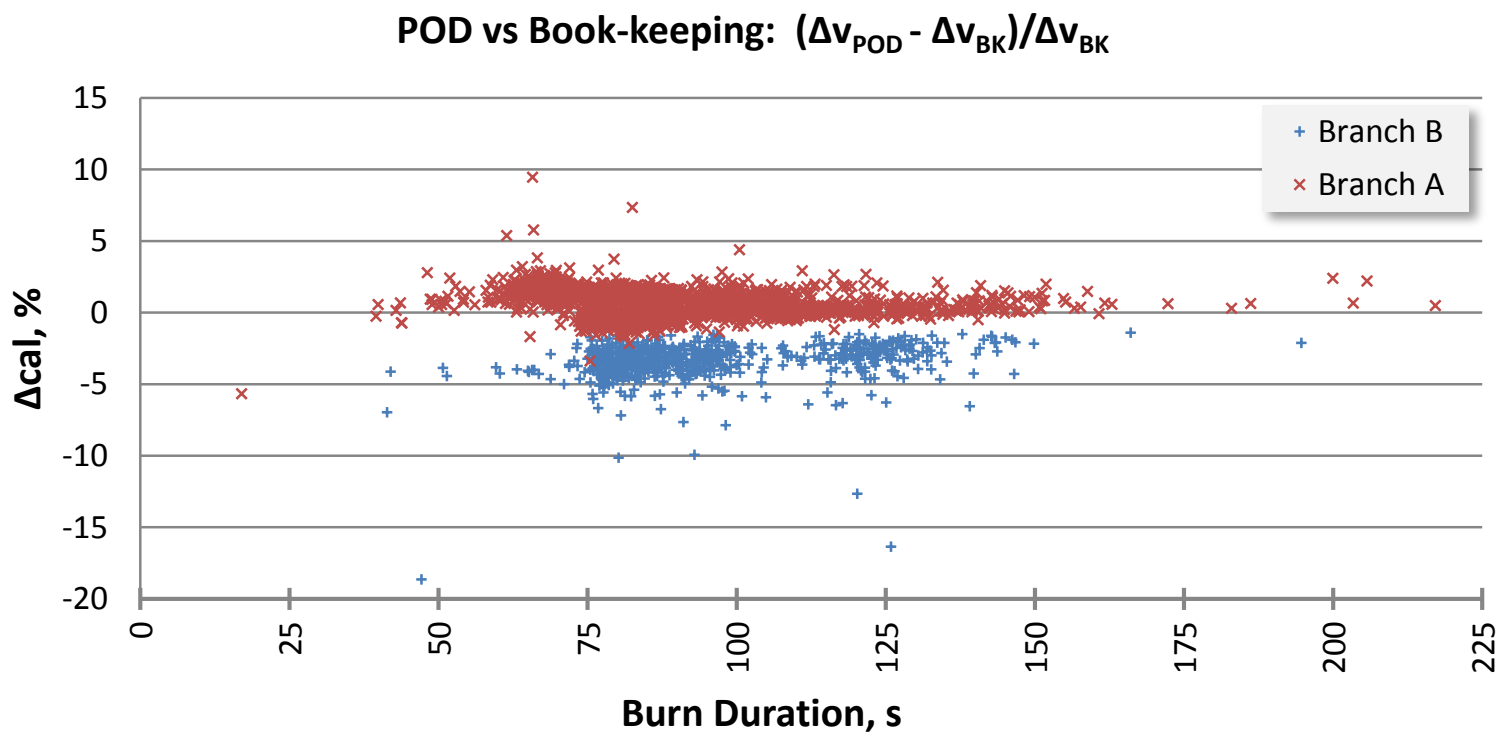

Figure 8: Dependency upon burn-duration. The variation in the Acal is larger for shorter burns because of increasing relative errors in both calibration methods. Most maneuvers with burn duration shorter than 75 seconds of branch A have a positive $\Delta$ cal. 


\section{Discussion of the achieved accuracy}

There are several uncertainties in both methods which have to be taken into account.

The attitude of TDX is varied continuously over the orbit to align the azimuth axis of the SAR instrument with the ground-track. Therefore, a small cross-coupling in normal direction exists and the main component in orbit tangential direction as determined in the POD method is smaller. The attitude offset is not a constant value but varies with geographic latitude. The maximum is reached over the equator and values $3.7^{\circ}$. This means that still $99.8 \%\left(\cos 3.7^{\circ}=0.998\right)$ of the thrust are reflected in the POD in the worst case.

The accuracy of the POD method was estimated to be of the order of 1.5 to $2.5 \%$ (see section I.C).

The thruster coefficients used in Eq. (1) are dependent upon the temperature. The thermal stability of the thruster brackets pronounced in I.C is not constantly fulfilled. Temperature variations reach up to $5^{\circ} \mathrm{C}$ due to eclipse seasons and limited power of the heaters. A variation of $5{ }^{\circ} \mathrm{C}$ in the gas temperature could change the specific impulse by about 1 second $(\triangleq 1.5 \%)$ according to the manufacturer. Furthermore, the pressure transducers are specified with an error range up to $\pm 0.5 \%$ (full scale output).

The reason for the larger $\Delta$ cal observed for branch B cannot be traced back to a single root cause. The mean deviation of $-3.4 \%$ is still very good.

\section{Future work}

The large data set allows for an independent determination of the thruster coefficients used in Eq. (1) which then can be compared with the pre-flight laboratory measurements. The $\Delta \mathrm{v}$ obtained in flight direction by each thruster pair is known via the POD. A straight line describes the thrust level as a function of pressure well.

$$
F_{P O D}=\frac{\Delta v_{P O D} M_{S C}}{\Delta t}=\sum_{i=1,2} a_{i}^{\prime}+b^{\prime}{ }_{i} \bar{p}
$$

A least squares fit was made for all maneuvers on branch A in flight direction. Figure 9 shows the thrust and the trend line for all of these maneuvers. The difference between the POD and book-keeping method - the latter with the newly determined coefficients (see table 3) - was calculated once more and is shown in Fig. 10. It is seen that the in situ calibration leads to almost perfect agreement of the two methods.

A similar evaluation for the other thruster pairs will be done in the near future. The new coefficients will be used for an even more precise maneuver planning.

An analysis of TAFF maneuvers will be done in the future as well.

Table 3. Calculated thruster coefficients. Slope and offset of the trend line in Fig. 9 are the sum of the new coefficients $\boldsymbol{a}^{\prime}$ and $\boldsymbol{b}^{\prime}$.

\begin{tabular}{|c|c|c|c|c|}
\hline Thruster & $\begin{array}{c}\text { Offset } \\
\boldsymbol{a}[\mathrm{mN}]\end{array}$ & $\begin{array}{c}\text { Calculated Offset } \\
\boldsymbol{a},[\mathrm{mN}]\end{array}$ & $\begin{array}{c}\text { Slope } \\
\boldsymbol{b}[\mathrm{mN} / \mathrm{bar}]\end{array}$ & $\begin{array}{c}\text { Calculated Slope } \\
\boldsymbol{b}\end{array}$ \\
\hline A1-plus $[\mathrm{mN} / \mathrm{bar}]$
\end{tabular}




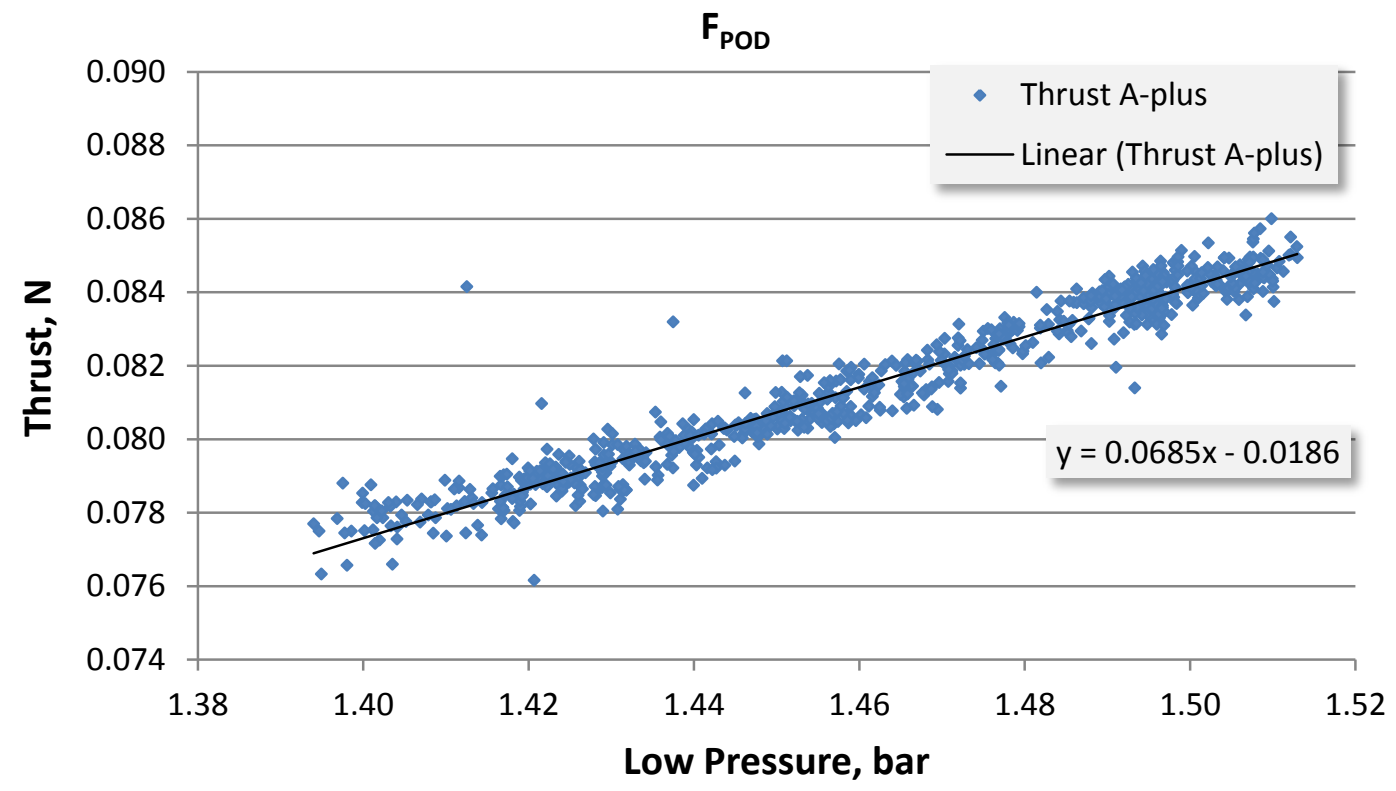

Figure 9: Thrust as a function of the low pressure. Shown are all maneuvers made with the $+x$ thrusters of branch A. Slope and offset of the trend line denote the sum of the coefficients of both thrusters.

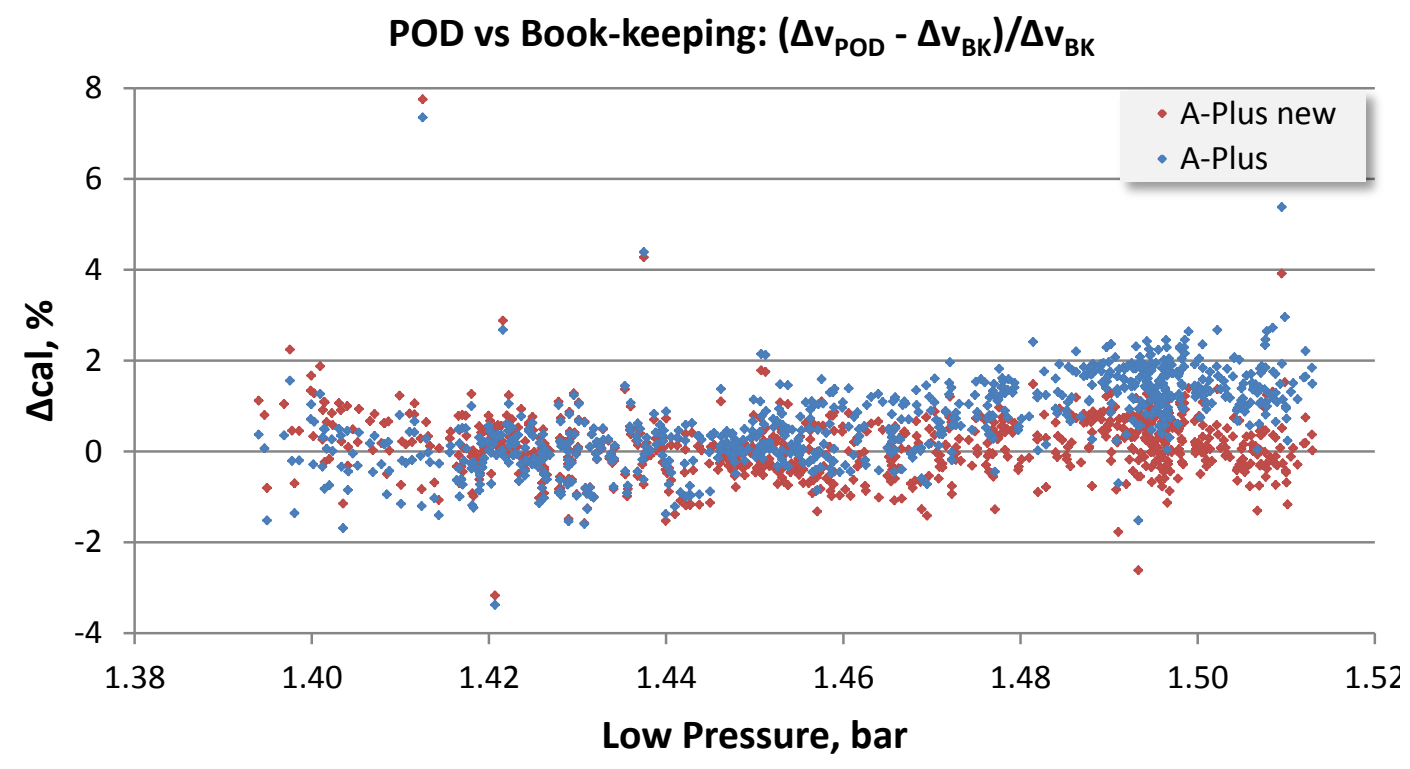

Figure 10: Comparison of the pre-launch thruster coefficients with the calibration in space. Shown is the $\triangle$ cal of POD and book-keeping method. Blue symbols show the results obtained with the coefficients from the manufacturer, red symbols with the newly calculated coefficients. Only maneuvers of branch A in flight-direction are shown. 


\section{Conclusion}

A calibration of 2360 cold gas maneuvers was made with two different methods - the book-keeping method on the one and by precise orbit determination on the other hand. The comparison shows remarkably small differences of only $0.6 \%$ on average for branch A and $-3.4 \%$ for branch B.

The influence of the low pressure and the burn duration was analyzed as well. A dependency upon the regulated pressure was found for both branches, which will allow for an even more precise prediction of the maneuver performance.

It has been shown that formation-keeping with the TDX cold gas propulsion system can be realized very accurately. On average the size of an executed maneuver differs from the planning by $1.5 \%$. The resulting execution error is only $0.0075 \mathrm{~cm} / \mathrm{s}$ for a typical tangential maneuver of $0.5 \mathrm{~cm} / \mathrm{s}$ size. The impact on the TDXTSX formation is estimated as a change of the relative semi-major axis of $13 \mathrm{~cm}$ and relative along-track drift of about $1 \mathrm{~m}$ per orbit. To put this in perspective, the relative atmospheric drag acting on the formation in $505 \mathrm{~km}$ altitude during moderate solar activity causes a change in the relative semi-major axis of about $15 \mathrm{~cm}$ per day. The dominating effect on the formation is the perturbation due to the oblateness of the Earth, which affects the cross-track separation. This perturbation is about 100 times larger than maneuver uncertainty or relative drag. Together with relative navigation errors a remarkable formation control accuracy of $5 \mathrm{~m}$ (RMS) in cross-track direction and $30 \mathrm{~m}(\mathrm{RMS})$ in along-track direction is achieved [5]. 


\section{Appendix A}

POD vs Book-keeping: $\left(\Delta \mathrm{v}_{\mathrm{POD}}-\Delta \mathrm{v}_{\mathrm{BK}}\right) / \Delta \mathrm{v}_{\mathrm{BK}}$
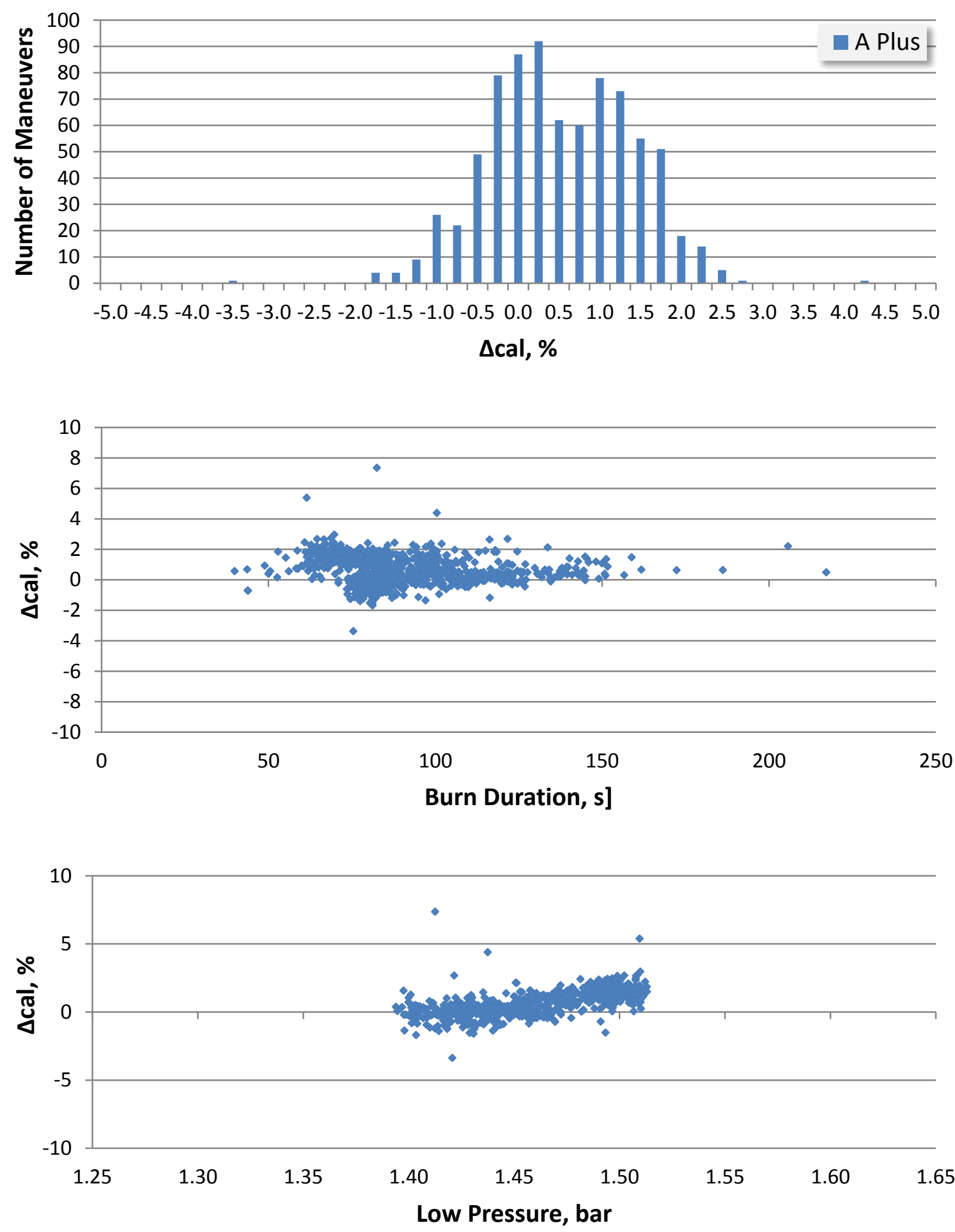

Figure 11: Maneuvers made with the $+x$ thrusters of branch A. 
POD vs Book-keeping: $\left(\Delta \mathrm{v}_{\mathrm{POD}}-\Delta \mathrm{v}_{\mathrm{BK}}\right) / \Delta \mathrm{v}_{\mathrm{BK}}$
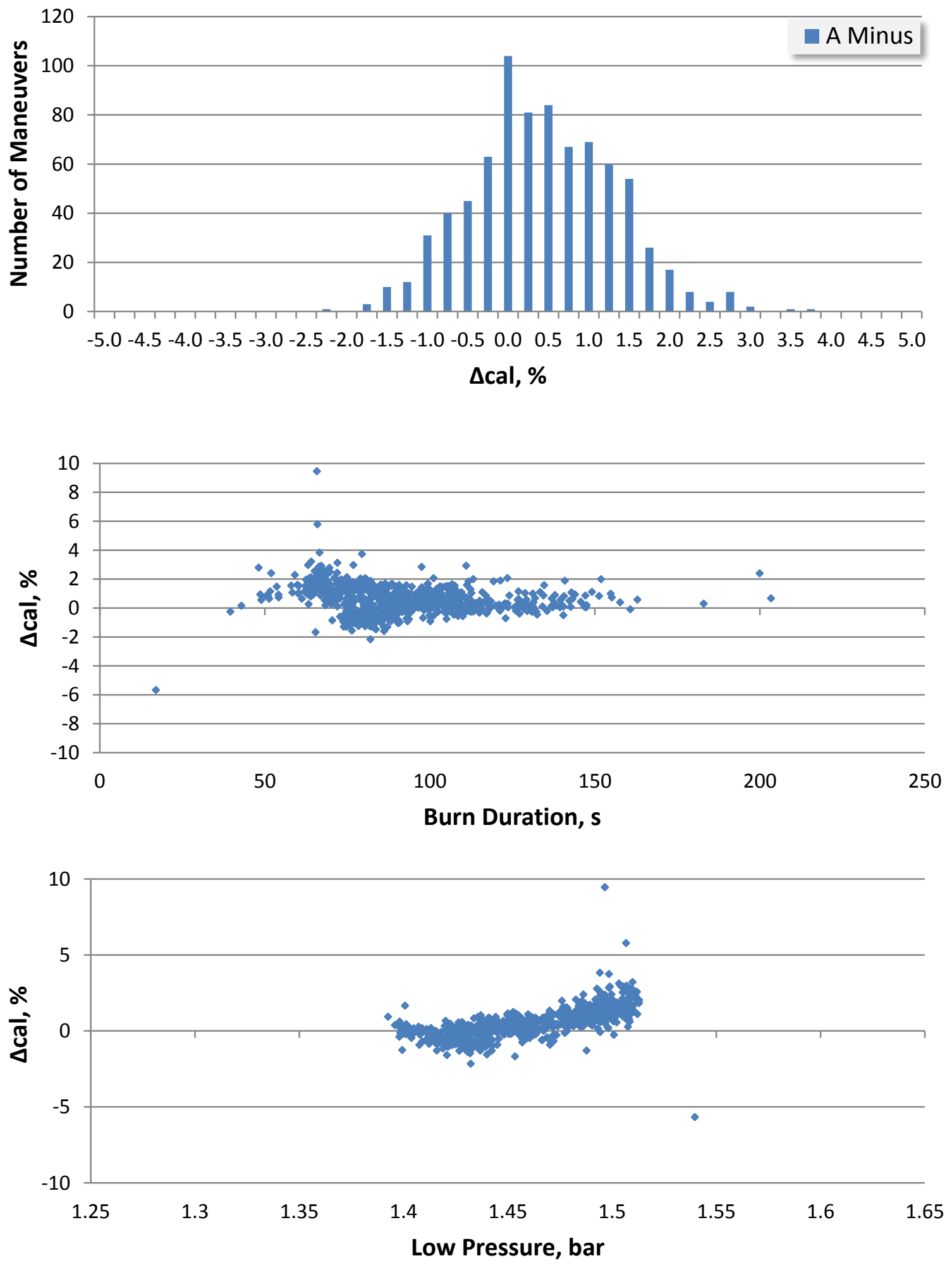

Figure 12: Maneuvers made with the -x thrusters of branch A. 
POD vs Book-keeping: $\left(\Delta v_{\mathrm{POD}}-\Delta \mathrm{v}_{\mathrm{BK}}\right) / \Delta \mathrm{v}_{\mathrm{BK}}$
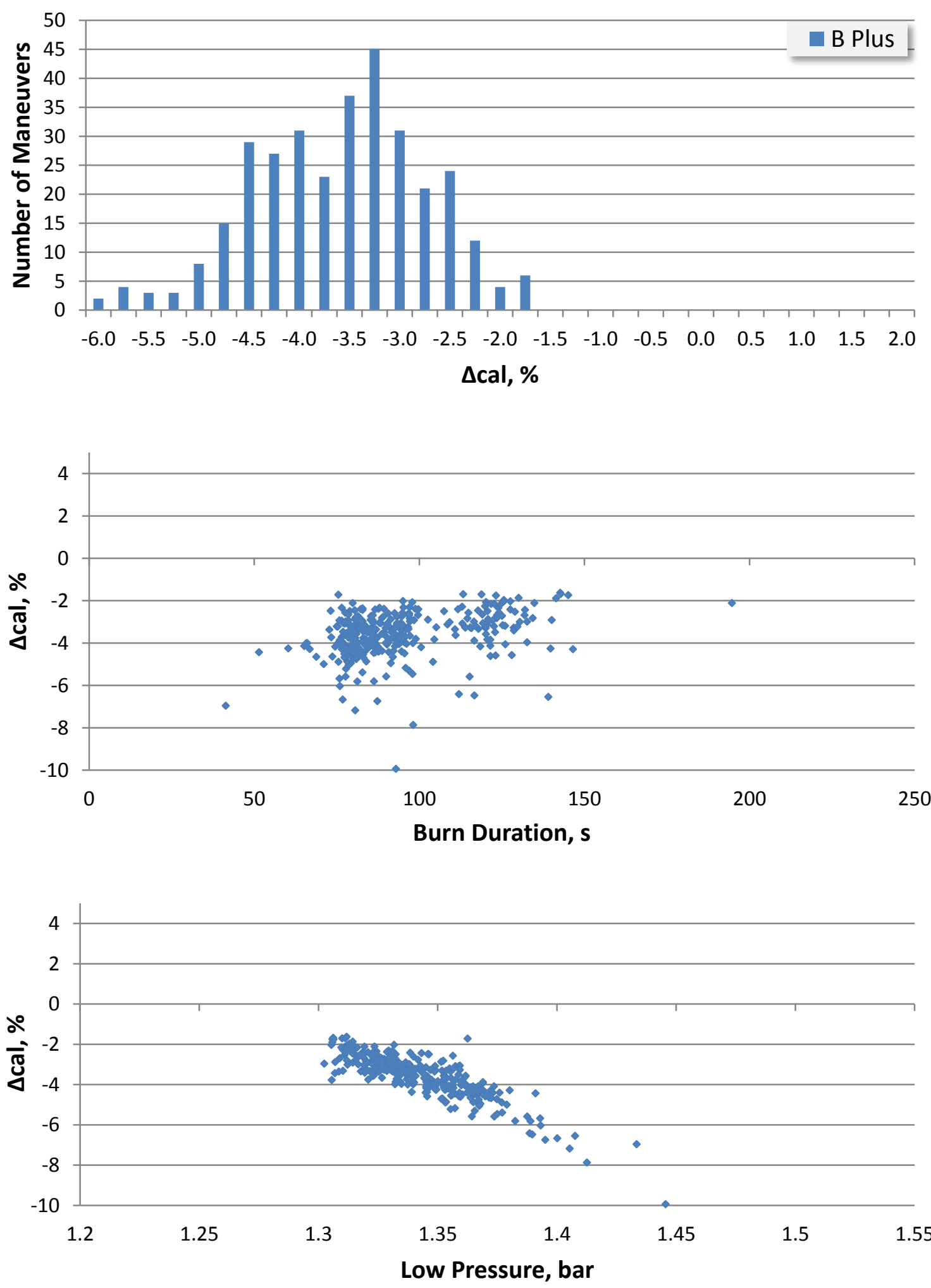

Figure 13: Maneuvers made with the $+x$ thrusters of branch $B$. 
POD vs Book-keeping: $\left(\Delta \mathrm{v}_{\mathrm{POD}}-\Delta \mathrm{v}_{\mathrm{BK}}\right) / \Delta \mathrm{v}_{\mathrm{BK}}$
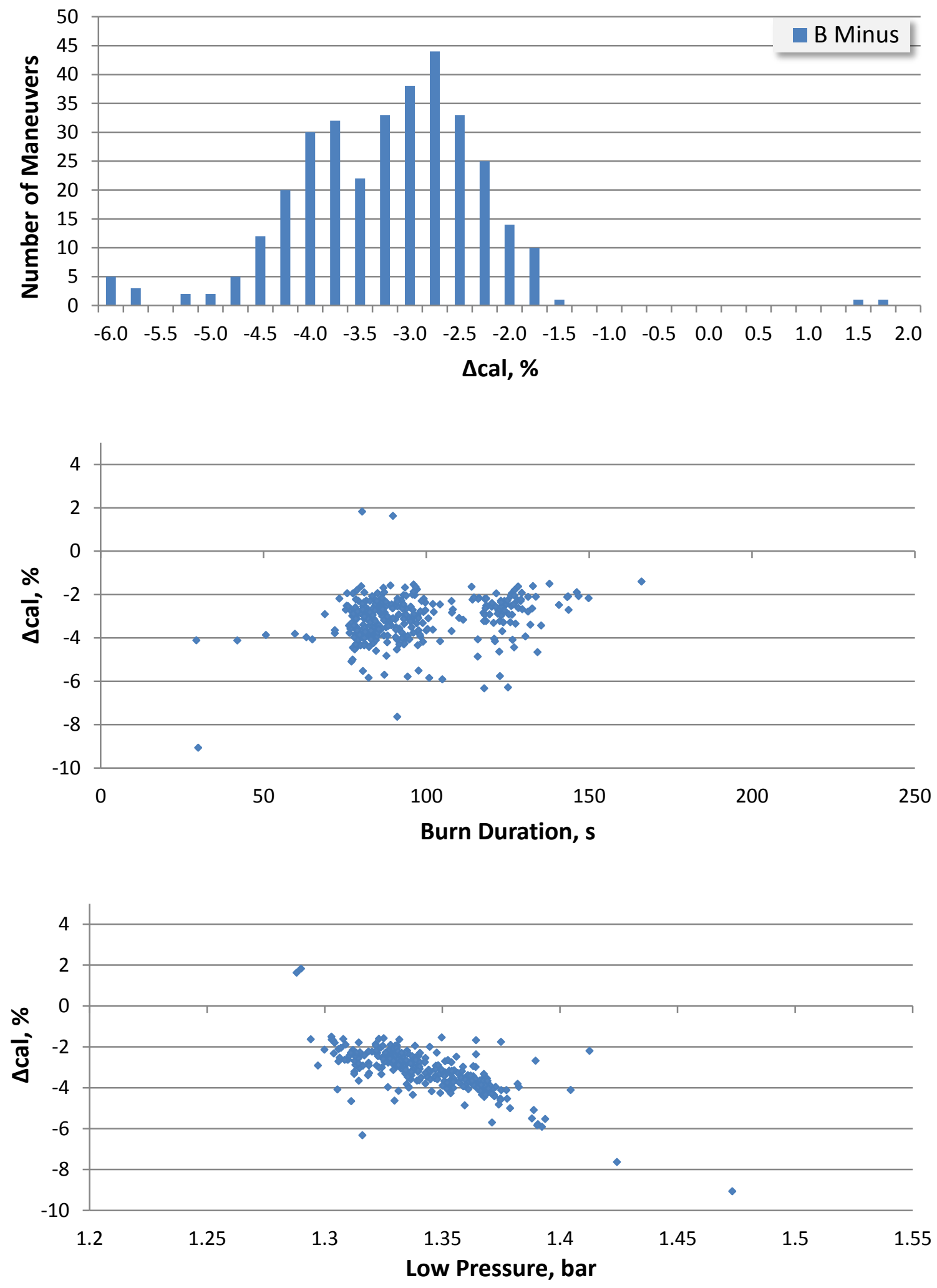

Figure 14: Maneuvers made with the -x thrusters of branch $B$. 


\section{Acronym List}

\begin{tabular}{ll} 
AOCS & Attitude and Orbit Control System \\
BK & Book-keeping \\
CGS & Cold Gas System \\
DEM & Digital Elevation Model \\
DLR & German Aerospace Center \\
GFZ & German Research Center for Geosciences \\
GHOST & GSOC High precision Orbit determination Software Tools \\
GRACE & Gravity Recovery And Climate Experiment \\
GSOC & German Space Operations Center \\
HPLV & High Pressure Latch Valve \\
HPT & High Pressure Transducer \\
IGOR & Integrated Geodetic Occultation and Ranging \\
ISP & Specific Impulse \\
LEOP & Launch and Early Orbit Phase \\
LPT & Low Pressure Transducer \\
MET & Mission Elapsed Time \\
POD & Precise Orbit Determination \\
PR & Pressure Regulator \\
SAR & Synthetic Aperture Radar \\
TAFF & Tandem Autonomous Formation Flight \\
TDX & TanDEM-X Satellite \\
TSX & TerraSAR-X Satellite \\
\hline
\end{tabular}

\section{Acknowledgments}

The authors wish to thank Fabiana Cossavella for her helpful comments and the critical reading of this article.

\section{References}

[1] Krieger, G., Zink, M., Bachmann, M., Bräutigam, B., Breit, H., Fiedler, H., Fritz, T., Hajnsek, I., Hueso Gonzalez, J., Kahle, R., König, R., Schättler, B., Schulze, D., Ulrich, D., Wermuth, M., Wessel, B., Moreira, A.; "TanDEM-X“; Distributed Space Missions for Earth System Monitoring Space Technology Library; Springer; P; 387-435. ISBN 978-14614-4540-1; 2012.

[2] Kahle, R., D'Amico, S.: “The TerraSAR-X Precise Orbit Control - Concept and Flight Results", International Symposium on Space Flight Dynamics, Laurel, MD, US, 2014 (http://issfd.org).

[3] Kahle, R., Schlepp, B., Meissner, F., Kirschner, M., Kiehling, R.; "TerraSAR-X/ TanDEM-X Formation Acquisition Analysis and Flight Results From Planning to Realization"; Journal of the Astronautical Sciences, 59 (3). ISSN 0021-9142, 2014.

[4] D'Amico, S., Montenbruck, O.; "Proximity Operations of Formation-Flying Spacecraft using eccentricity/inclination vector separation", Journal of Guidance, Control, and Dynamics, 2006.

[5] Kahle, R., Schlepp, B., Aida, S., Kirschner, M., Wermuth, M.: "Flight Dynamics Operations of the TanDEM-X Formation"; SpaceOps conference 2012, Stockholm, Sweden, 2012.

[6] Montenbruck, O., van Helleputte, T., Kroes, R., "Reduced dynamic orbit determination using GPS code and carrier measurements", Aerospace Science and Technology, 9 (3), pp. 261-271. DOI: 10.1016/j.ast.2005.01.003, 2005.

[7] Wermuth, M., Hauschild, A., Montenbruck, O., Kahle, R.: "TerraSAR-X precise orbit determination with real-time GPS ephemerides", Advances in Space Research, 50 (5), pp. 549-559. DOI: 10.1016/j.asr.2012.03.014, 2012.

[8] Yoon, Y., Montenbruck, O., Kirschner, M.: "Precise maneuver calibration for remote sensing satellites", 19th International Symposium on Space Flight Dynamics, Kanazawa, Japan, 2006. (http://issfd.org).

[9] Ardaens, J.S., Kahle, R., Schulze, D., "In-Flight Performance Validation of the TanDEM-X Autonomous Formation Flying System", 5th International Conference on Spacecraft Formation Flying Missions and Technologies, Munich, Germany, 2013. 